\title{
Critical evaluation of ivabradine for the management of chronic stable angina
}

\author{
This article was published in the following Dove Press journal: \\ Research Reports in Clinical Cardiology \\ 2 September 201 I \\ Number of times this article has been viewed
}

\author{
Waqas Khan \\ Jeffrey S Borer \\ Division of Cardiovascular Medicine \\ and the Department of Medicine, \\ State University of New York \\ Downstate Medical Center and \\ College of Medicine, Brooklyn \\ and New York, NY, USA
}

\begin{abstract}
Angina pectoris is the most common symptom of coronary artery disease (CAD). Angina results from an imbalance between myocardial oxygen supply and demand. Heart rate (HR) reduction can beneficially alter both elements of this imbalance by increasing diastolic filling time and reducing myocardial oxygen demand. Therefore, HR reduction is an accepted approach to angina prevention. $\beta$-blockers, calcium-channel blockers, and long-acting nitrates are currently the cornerstones in prevention and management of stable angina. However, use of these treatments may be limited by adverse effects or development of tolerance. Thus, additional approaches to angina prevention may be useful for many patients with CAD. The discovery of the f-channel and the resulting current, $I_{\mathrm{f}}$, that modulates the rate of spontaneous diastolic depolarization of sinoatrial nodal (SAN) myocytes led to the study of these channels as targets for lowering HR. This resulted in the development of a novel agent, ivabradine, a selective and specific $I_{\mathrm{f}}$ inhibitor. Ivabradine slows the slope of diastolic depolarization of the action potential in the SAN cells, decreasing HR at rest and during exercise, but has no other cardiovascular effects. In different subpopulations with chronic stable angina, ivabradine markedly improves exercise capacity and significantly decreases the number of ambient angina attacks. In a post-hoc analysis of the BEAUTIFUL trial (morBidity-mortality EvAlUaTion of the $I_{\mathrm{f}}$ inhibitor ivabradine in patients with coronary disease and left-ventricULar dysfunction), ivabradine also reduced mortality, myocardial infarctions, and heart failure hospitalizations among patients with angina. To date, the drug has been well tolerated; transient visual disturbances and bradycardia are the only potentially important, though relatively infrequent, concerns and are readily reversible with drug cessation. This article will review and critically evaluate the data supporting use of ivabradine in patients with CAD and angina, both in preventing the symptom and, potentially, in altering the natural history of CAD.
\end{abstract}

Keywords: coronary artery disease, cardiovascular pharmacology, clinical trials

\section{Introduction: pathophysiology and management issues in chronic stable angina and the role of heart rate}

Typical angina pectoris is the most common presenting symptom of coronary artery disease (CAD), itself the single most common cause of death worldwide. ${ }^{1}$ Typical angina is a symptom consisting of central chest discomfort stimulated by physical exertion or emotional stress, which is relieved within a relatively short interval after stopping the inciting stress and/or after administering sublingual nitroglycerin. Angina results from an imbalance between myocardial perfusion and myocardial metabolic demands. ${ }^{2,3}$ Heart rate (HR) reduction can alter both elements of this 
imbalance beneficially by increasing diastolic filling time (in turn, increasing myocardial perfusion) and reducing myocardial oxygen demand. Therefore, HR reduction is a characteristic of several existing anti-anginal, antiischemic therapies and is accepted as a useful approach to this problem. This article will focus on evaluation of a novel agent, ivabradine, which specifically and solely acts by slowing HR, now approved for marketing in Europe and several other parts of the world but which has not yet been submitted to the US Food and Drug Administration (FDA) for consideration.

Angina is a symptom, not a disease, and it has many causes other than CAD. However, when CAD is the cause, angina has an important negative impact on quality of life. ${ }^{4,5}$ Thus, prevention of angina due to CAD can provide important benefits to afflicted patients. Moreover, the presence of angina can signal the need for additional therapy to prevent or minimize the major morbid and lethal sequelae of CAD. Indeed, despite current approaches, the burden of $\mathrm{CAD}$ remains high across the world and is projected to be the leading cause of death for at least the next 20 years. ${ }^{6}$ The Framingham Heart Study has shown that the lifetime risk of CAD for patients aged 40 years is $31 \%$ for females and $48 \%$ for males. ${ }^{7}$ In this context, it is of interest to determine whether therapeutic modalities that can relieve or prevent angina can also beneficially affect the natural history of CAD. Until the development of ivabradine, among antianginals only nicorandil had been tested for such benefits. However, experimentally, HR has been directly associated with the progression of coronary atherosclerosis ${ }^{8-10}$ and, clinically, HR is directly related to the likelihood of disrupting preexisting atherosclerotic plaque. ${ }^{11}$ Therefore, it is possible that HR-slowing anti-anginal therapies may have a particular advantage for patients with angina due to CAD by reducing major morbid and lethal ischemic event risk.

Several pharmacological treatments and mechanical interventions (eg, coronary angioplasty, coronary artery bypass grafting [CABG]) can prevent or reduce the frequency of stable angina. Conventional pharmacological management includes $\beta$-blockers, calcium-channel blockers, organic nitrates, and agents with novel pharmacological effects that may directly affect various aspects of myocardial metabolism. Some of these, including $\beta$-blockers and certain verapamil-type calcium-channel blockers, reduce HR. However, all conventional HR-lowering drug therapies also have other pharmacological effects directly on the cardiovascular system (eg, on systolic and/or diastolic myocardial function and vasodilatation). In addition, of course, all drugs can have noncardiovascular "off-target" effects that may be intolerable or even deleterious in some patients. ${ }^{12-15}$ Finally, a substantial proportion of patients will not respond to any specific anti-anginal drug; for example, approximately one-fifth of patients with chronic stable angina do not respond to $\beta$-blocker therapy. ${ }^{16}$ Indeed, despite current multidrug and multimodality therapy, one-third to one-half of patients with chronic stable CAD continue to manifest angina. ${ }^{17}$ Thus, additional approaches to angina prevention have potentially important utility for many patients with CAD.

The discovery of the f-channel and the resulting current, $I_{\mathrm{f}}$, that modulates the rate of spontaneous diastolic depolarization of sinoatrial nodal (SAN) myocytes, led to the study of these channels as potential targets for specific lowering of HR. ${ }^{18,19}$ This resulted in the development of ivabradine, a selective and specific $I_{\mathrm{f}}$ inhibitor. Ivabradine slows the slope of diastolic depolarization of the action potential in the SAN cells, decreasing HR at rest and during exercise.

In SAN and certain other cardiac tissues (atrioventricular [AV] node, Purkinje fibers), electrophysiological pacemaking (stimulation of generalized cardiac depolarization and contraction) is the result of the interplay of several ionic currents, pumps, and exchangers that contribute to generation of spontaneous repetitive action potentials. The myocytes of the SAN are the primary pacemaker cells of the heart and therefore control HR. ${ }^{2,20-23}$ Of the several mechanisms providing the cellular and molecular properties necessary for intrinsic pacemaking to occur in SAN cells, an important role is played by the so called "funny current $\left(I_{\mathrm{f}}\right)$." ${ }^{18}$ The $I_{\mathrm{f}}$ current was first described more than 30 years ago by DiFrancesco and colleagues; ${ }^{24}$ the current was denoted as "funny" (f) because it has unusual properties compared with other cardiac currents, including mixed permeability of the f-channel to sodium and potassium ions, activation by hyperpolarization and intracellular cyclic adenosine monophosphate (cAMP), low single-channel conductance, and slow kinetics. ${ }^{25}$ The $I_{\mathrm{f}}$ current is modulated by activity of the autonomic nervous system. A rise in intracellular cAMP under the influence of $\beta$-receptor stimulation results in an increase in the $I_{\mathrm{f}}$ current and the diastolic depolarization slope, which then leads to a decrease in the duration of diastole, producing an increase in HR. The opposite occurs when the muscarinic receptors are stimulated: diastolic duration increases and HR decreases. ${ }^{26-29}$

The f-channels responsible for the $\mathrm{I}_{f}$ current are part of the hyperpolarization-activated, cyclic nucleotide-gated (HCN) channel family of which four distinct isoforms 
are known (HCN 1-4). These isoforms vary in terms of their properties and distribution in several different tissues, including the retina, brain, and heart. ${ }^{25,30,31}$ Of these isoforms, the HCN4 channel is found in the heart and is active in the SAN. HCN4 channels found in the Purkinje fibers and the AV node are inactive under normal physiological conditions (but, based on experimental evidence, may operate under pathological conditions such as heart failure $\left.{ }^{20}\right)$.

\section{Pharmacology of ivabradine}

Inhibition of the $I_{\mathrm{f}}$ current is useful in reducing $\mathrm{HR}$ and thus in preventing angina pectoris and possibly other manifestations of $\mathrm{CAD}^{20,21,32}$ and heart failure. Ivabradine slows the HR by specifically binding to the f-channels on the intracellular side of the plasma membrane of the SAN cells, thereby selectively inhibiting the $I_{\mathrm{f}}$, decreasing the slope of SAN diastolic depolarization, and decreasing HR both at rest and during exercise. ${ }^{12,13}$

Ivabradine inhibits the f-channel in the open phase when channels deactivate upon depolarization and is relieved during hyperpolarization in the closing phase, thereby acting as an open-channel blocker (Figure 1). ${ }^{33}$ Therefore, the effect of ivabradine is "use-dependent", ie, the greater the time that the channels are open (ie, the higher the HR), the greater the effect of the drug in slowing HR; ${ }^{12,13,34,35}$ conversely, the drug has diminished HR slowing effects at slower HR, providing a measure of safety against excessive HR slowing.

\section{Pharmacokinetics}

Under physiological conditions, ivabradine (as the S-enantiomer) is rapidly released from tablets and is highly water soluble $(>10 \mathrm{mg} / \mathrm{mL})$. There is no bioconversion of the drug, demonstrable in vivo. The $\mathrm{N}$-desmethylated derivative of ivabradine has been identified as the main active metabolite in humans. ${ }^{36}$

\section{Absorption and bioavailability}

After oral administration, ivabradine is rapidly and almost completely absorbed, reaching a peak plasma level in about 1 hour under fasting conditions. The absolute bioavailability of the film-coated tablets is approximately $40 \%$, due to a first-pass effect in the gut and liver. Food delays absorption by approximately 1 hour, and increases plasma exposure by $20 \%-30 \%$. The intake of the tablet during meals is recommended in order to decrease intra-individual variability in exposure. ${ }^{36}$

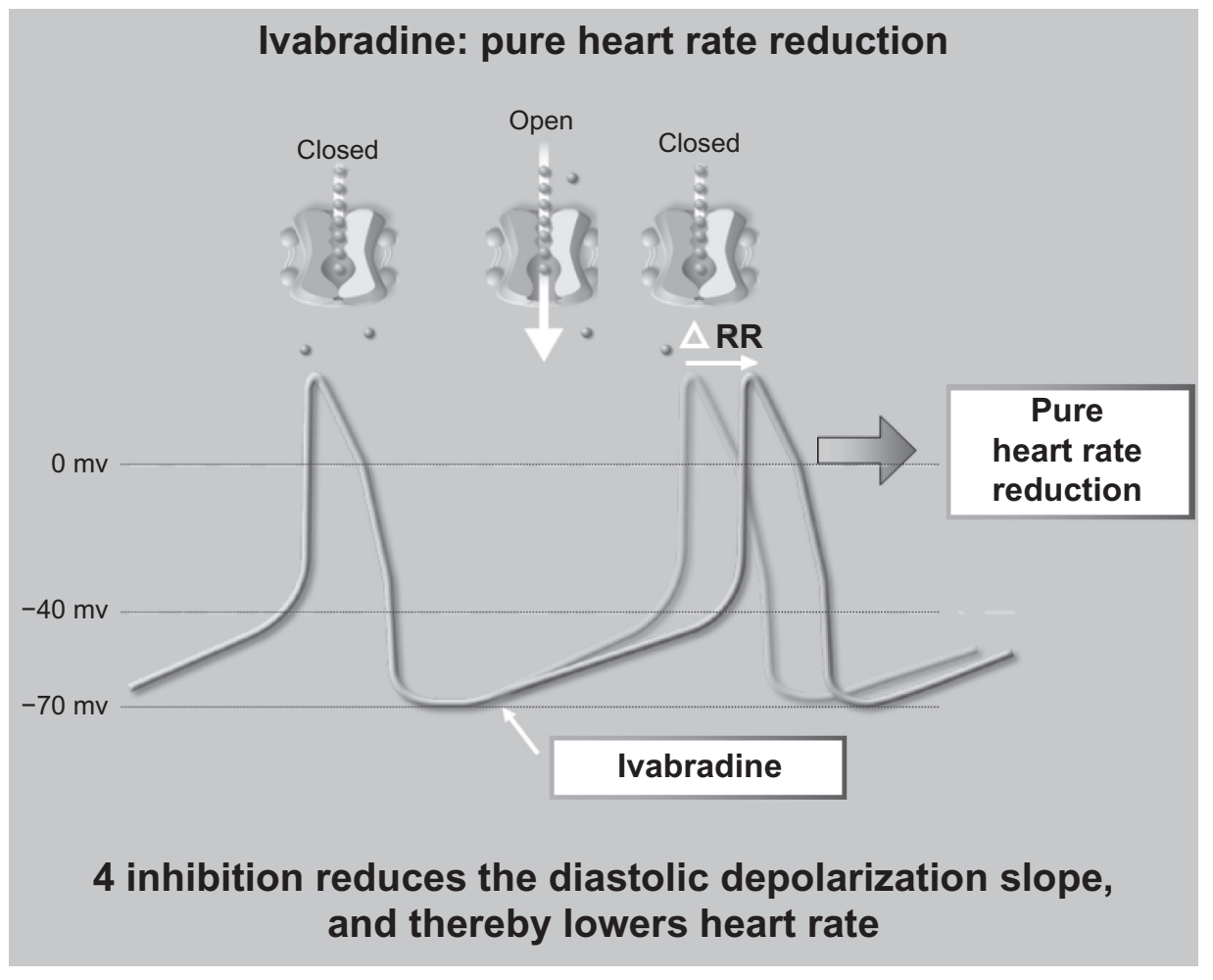

Figure I Pharmacological basis of ivabradine effects.

Note: Reproduced from César ${ }^{32}$ with permission of the publisher.

Abbreviation: RR, interval between successive ECG $R$ waves. 


\section{Distribution}

Ivabradine is approximately $70 \%$ plasma protein bound. At recommended dose of $5 \mathrm{mg}$ twice daily, the maximum plasma concentration following chronic administration is $22 \mathrm{ng} / \mathrm{mL}$. The average plasma concentration is $10 \mathrm{ng} / \mathrm{mL}$ at steady-state. ${ }^{36}$

\section{Biotransformation}

Ivabradine is extensively metabolized by the liver and the gastrointestinal tract by oxidation through cytochrome P450 3A4 (CYP3A4) only. The major active metabolite is the N-desmethylated derivative (S 18982) with an exposure about $40 \%$ of that of the parent compound. The metabolism of this active metabolite also involves CYP3A4. Ivabradine has low affinity for CYP3A4, shows no clinically relevant CYP3A4 induction or inhibition, and is therefore unlikely to modify CYP3A4 substrate metabolism or plasma concentrations. Conversely, potent inhibitors and inducers of the enzyme may substantially affect ivabradine plasma concentrations. ${ }^{36}$

\section{Elimination}

Ivabradine is eliminated with a main half-life of 2 hours ( $70 \%-75 \%$ of the area under the curve $[\mathrm{AUC}]$ ) in plasma and an effective half-life of 11 hours. The total clearance is about $400 \mathrm{~mL} / \mathrm{min}$ and the renal clearance is about $70 \mathrm{~mL} / \mathrm{min}$. Excretion of metabolites occurs to a similar extent via feces and urine. About $4 \%$ of an oral dose is excreted unchanged in urine. ${ }^{36}$

\section{Linearity/Non linearity}

The kinetics of ivabradine are linear over an oral dose range of $0.5-24 \mathrm{mg}$.

\section{Special populations}

No pharmacokinetic differences have been observed between elderly ( $\geq 65$ years) or very elderly patients ( $\geq 75$ years) and the overall population.

The impact of renal impairment (creatinine clearance from 15 to $60 \mathrm{~mL} / \mathrm{min}$ ) on ivabradine pharmacokinetics is minimal, consistent with the relatively small contribution of renal clearance (about 20\%) to total elimination for both ivabradine and S 18982. In patients with mild hepatic impairment (Child Pugh score up to 7) AUC of unbound ivabradine and S 18982 are about 20\% higher than in subjects with normal hepatic function. Data are insufficient to draw conclusions in patients with moderate hepatic impairment. No data are available in patients with severe hepatic impairment. ${ }^{36}$

\section{Pharmacokinetic/pharmacodynamic (PK/PD) relationship}

HR decreases almost linearly with increasing ivabradine and S 18982 plasma concentrations for doses of up to $15-20 \mathrm{mg}$ twice daily. At higher doses, the decrease in HR is no longer proportional to ivabradine plasma concentrations and tends to reach a plateau. High exposures to ivabradine that may occur when ivabradine is given in combination with strong CYP3A4 inhibitors may result in an excessive decrease in HR (largely limited by use dependence); this effect is less marked when moderate CYP3A4 inhibitors are employed. ${ }^{36}$

\section{Clinical effects: anti-anginal/ anti-ischemic efficacy}

The anti-anginal and anti-ischemic efficacy of ivabradine has been confirmed in patients with stable angina in clinical studies comparing it either with placebo or with active antianginal agents.

\section{Versus placebo and dose response}

The clinical efficacy and safety of ivabradine were evaluated by Borer et al. ${ }^{37}$ In this study, during a 2-week double-blind, parallel arm, dose-ranging phase, doses of $2.5 \mathrm{mg}$ bid, $5 \mathrm{mg}$ bid, and $10 \mathrm{mg}$ bid were compared with placebo bid (Figure 2). Resting HR at trough of drug activity decreased relative to placebo in all three active treatment groups $(P=0.05)$. Maximal HR during exercise decreased in parallel. Time to $1 \mathrm{~mm}$ ST depression during exercise tolerance testing (ETT) increased with ivabradine treatment. The change was significant relative to placebo in the $5 \mathrm{mg}$ bid and $10 \mathrm{mg}$ bid groups; a significant dose-response relation was seen across all doses. Time to limiting angina nominally increased at all doses but reached significance only for $10 \mathrm{mg}$ bid ivabradine. Ivabradine-treated patients showed

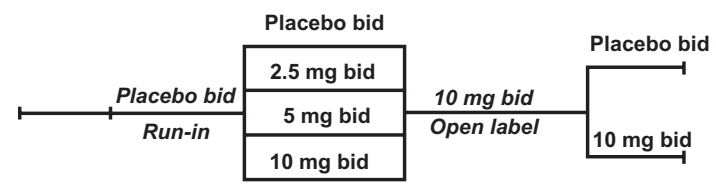

\begin{tabular}{|c|c|c|c|c|}
\hline 2 to $7 d$ & 1 week & 2 weeks & $2-3$ months & 1 week \\
\hline & & \multicolumn{2}{|c|}{$\Uparrow \uparrow$} & $\uparrow$ \\
\hline ET & & & & ETT \\
\hline
\end{tabular}

Figure 2 Study design of placebo-controlled clinical trial of ivabradine as monotherapy for prevention of chronic stable angina.

Note: Reproduced from Borer et $\mathrm{al}^{37}$ with permission of the publisher.

Abbreviation: ETT, exercise tolerance testing; bid, twice daily. 
significant dose-dependent $(P=0.002)$ increase in total work performed during upright bicycle ETT $(P=0.019)$. There was significant superiority of $10 \mathrm{mg}$ bid ivabradine versus placebo for total work performed. Angina attacks and consumption of short-acting nitrates nominally were reduced by ivabradine during the double-blind dose ranging phase, although these changes did not reach statistical significance.

At the end of the 2-week double-blind phase, all patients who chose to continue in the trial were titrated to $10 \mathrm{mg}$ bid of ivabradine in open-label fashion. Compared with initial treatment with placebo or with lower doses of ivabradine, exercise tolerance nominally improved at the higher dose. At the end of 2 or 3 months of open-label extension, patients were randomly assigned in blinded fashion to immediate withdrawal to placebo or maintenance of ivabradine $10 \mathrm{mg}$ bid. After 1 week, ETT was again performed and demonstrated significantly greater exercise tolerance with ivabradine than with placebo. Upon withdrawal, no evidence of pharmacological tolerance or rebound was observed.

The long-term clinical efficacy of two doses of ivabradine was evaluated by Lopez et al. ${ }^{38}$ Ivabradine was administered in doses of 5 and $7.5 \mathrm{mg}$ bid in patients with concomitant antianginal medications. HR reduction and antianginal efficacy were maintained for the 12-month duration of therapy.

\section{Versus $\beta$-blockade}

Ivabradine was compared directly with a $\beta$-blocker, atenolol, in the INternatIonal TrIal of the AnTi-anginal effects of IVabradinE compared to atenolol (INITIATIVE), a randomized double-blinded, parallel-group trial involving 144 centers in 21 countries (Figure 3 ). ${ }^{3}$ Ivabradine and atenolol therapy were begun at $5 \mathrm{mg}$ bid and $50 \mathrm{mg}$ daily (with a

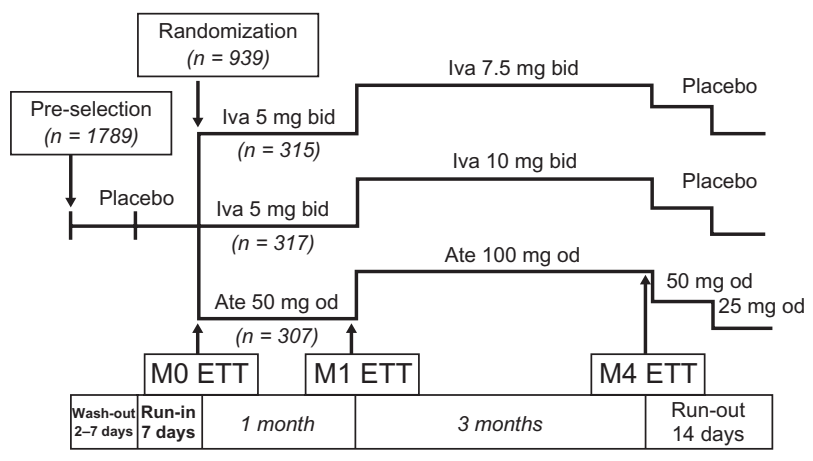

Figure 3 Study design of active controlled non-inferiority trial of ivabradine versus atenolol for prevention of chronic stable angina.

Note: Reproduced from Tardif et $\mathrm{al}^{3}$ with permission of the publisher.

Abbreviations: Ate, atenolol; bid, twice daily; ETT, exercise tolerance testing; Iva, ivabradine; M, month; od, once daily. second placebo tablet), respectively, and then titrated at 1 month of therapy to $7.5 \mathrm{mg}$ bid or $10 \mathrm{mg}$ bid versus $100 \mathrm{mg}$ daily (with second placebo tablet). These doses were selected to achieve relatively similar effects on HR with the two drugs, and indeed, no statistically significant differences in HR were observed, though atenolol nominally lowered HR slightly more than ivabradine for each comparison. The results of INITIATIVE showed that ivabradine effects on treadmill exercise tolerance and time to ST-segment depression at trough of drug concentration were not inferior to those of atenolol at the doses employed $(P<0.0001)$. However, though not statistically significant, after 4 months of treatment, all ETT parameters (time to limiting angina, time to angina onset, and time to 1-mm ST-segment depression) tended to have improved more with ivabradine than with atenolol. For both drugs, compared with pre-treatment, by the end of the trial the number of ambient angina attacks and the frequency of short-acting nitrate use decreased by almost two-thirds. Though differences in HR and ETT outcomes between the two drugs were not statistically significant themselves, the nominal differences achieved (slightly lower HR with atenolol, slightly greater exercise tolerance with ivabradine) resulted in a significantly greater increase in exercise capacity per beat reduction in HR with ivabradine than with atenolol. This might be attributable to ivabradine's lack of negative inotropic, lusitropic, peripheral vascular, or coronary vasoconstrictor effects compared with $\beta$-blockade. ${ }^{39}$

The safety of atenolol versus ivabradine was not rigorously compared in the INITIATIVE trial because twothirds of the patients had previously used $\beta$-blockers and were found to tolerate these drugs. Furthermore, patients with known contraindications or intolerance to atenolol were specifically excluded from the study.

Long-term efficacy and safety of ivabradine versus atenolol was assessed in a noninferiority trial of ivabradine $10 \mathrm{mg}$ bid (212 patients) versus atenolol $100 \mathrm{mg}$ daily (106 patients). ${ }^{40}$ Patients were followed for 1 year. Primary efficacy measures include total angina attacks by diary and short-acting nitrate consumption. Ambient angina attacks were decreased by 1.3 per week with ivabradine versus 0.8 with atenolol.

In a review article which explored the safety and efficacy of ivabradine compared with atenolol, ${ }^{41} \mathrm{HR} \leq 55$ beats per minute (bpm) was reported in 3.2\% (53 of 1651) of patients receiving therapeutic doses of ivabradine $(5 \mathrm{mg}$ or $7.5 \mathrm{mg}$ twice daily), compared with $5.1 \%$ (21 of 408) of patients treated with atenolol. The incidence of sinus bradycardia $<40$ bpm was $0.5 \%$ with ivabradine $7.5 \mathrm{mg}$ 
twice daily, and $1.7 \%$ with atenolol $100 \mathrm{mg}$ daily; no such cases were reported with ivabradine $5 \mathrm{mg}$ twice daily. Only $0.2 \%$ of patients experienced clinically important symptomatic bradycardia compared with $0.4 \%$ in the atenolol group, and $<1 \%$ of patients withdrew from therapy (ivabradine only) because of untoward bradycardia. In patients with HR $<45$ bpm on treatment, bradycardia-related symptoms such as hypotension and dizziness were not different in patients treated with ivabradine and patients treated with atenolol, whereas syncope occurred only in the atenolol arm. Dyspnea and fatigue occurred more commonly with atenolol.

\section{Versus amlodipine}

The anti-anginal and anti-ischemic efficacy of ivabradine (at two different doses, $7.5 \mathrm{mg}$ bid and $10 \mathrm{mg}$ bid) has been compared with amlodipine $10 \mathrm{mg}$ daily in patients with stable angina (Figure 4). In a large multicenter, international, double-blind, randomized, parallel-group trial among patients with stable angina by Ruzyllo et al, ${ }^{42}$ at trough of drug concentration, ivabradine was noninferior to amlodipine in improving exercise tolerance as well as increasing time to angina onset, time to limiting angina and time to $1 \mathrm{~mm}$ ST-segment depression. Ivabradine also was noninferior to amlodipine $(P<0.001)$ in preventing angina attacks and limiting nitrate use, though ivabradine caused greater reduction of rate-pressure product, surrogate for myocardial oxygen consumption, than amlodipine. Safety was indistinguishable between the two drugs.

\section{Combination therapy}

Combination therapy is widely used in clinical practice to achieve acceptable control of angina. Conventional

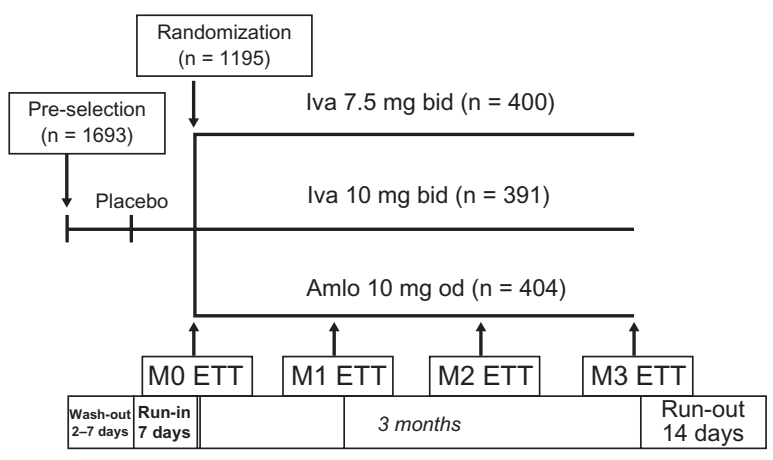

Figure 4 Study design of active-controlled non-inferiority trial of ivabradine versus amlodipine for prevention of chronic stable angina $(n=1195)$.

Note: Study design information dervied from Ruzyllo et al. ${ }^{42}$

Abbreviations: Amlo, amlodipine; bid, twice daily; ETT, exercise tolerance testing; Iva, ivabradine; M, month; od, once daily. anti-angina therapies often do not completely prevent symptoms, and for example, about $20 \%$ of patients do not respond adequately to $\beta$-blocker treatment alone. ${ }^{21}$ Adding a second agent is therefore the logical next step in order to achieve better control, ${ }^{15,43}$ a benefit that is difficult to demonstrate in clinical trials but has been sufficiently supported by such trials that it is standard practice. Ivabradine has been tested, and found effective, versus placebo on a background of other conventional anti-anginal drugs in several studies. ${ }^{2,44,45,64}$ Importantly, as demonstrated in several large studies performed for other purposes, the combination of multiple approved anti-anginal drugs with ivabradine also is acceptably safe for chronic use. ${ }^{45,46}$

\section{With atenolol}

In the Anti-anginal and Anti-ischaemic Effects of the $I_{\mathrm{f}}$ Current Inhibitor Ivabradine in Patients with Stable Angina Pectoris Receiving Standard Doses of Beta-Blockers (ASSOCIATE) study, ivabradine was compared with placebo in 889 patients with stable angina on a background of $\beta$-blockade with $50 \mathrm{mg}$ daily of atenolol. ${ }^{44}$ Compared with placebo, the addition of ivabradine at doses of $5 \mathrm{mg}$ bid for 2 months, then $7.5 \mathrm{mg}$ bid for 2 additional months, significantly improved all parameters of exercise capacity including total exercise duration, time to 1-mm ST depression, angina onset, and limiting angina, all at trough of drug activity. Ivabradine treatment produced dose-dependent reduction in $\mathrm{HR}$, both at rest $(-8.7 \pm 9.8 \mathrm{bpm}, 95 \%$ confidence interval $[\mathrm{CI}]-8.7$ to -6.2$)$ and at the peak exercise $(-11.3 \pm 13.2 \mathrm{bpm}, 95 \%$ CI 12.4 to -9.1$)$. The combination of atenolol and ivabradine was also well tolerated. Only $1.1 \%$ of patients withdrew owing to sinus bradycardia in the ivabradine group compared with none in the placebo group; $1.8 \%$ of participants in the ivabradine group reported visual symptoms (reversible - see below) versus $0.9 \%$ in the placebo group. No other major adverse effects were associated with ivabradine.

\section{With amlodipine}

The anti-anginal and anti-ischemic efficacy of ivabradine in addition to amlodipine was assessed in a 3-month trial ${ }^{40}$ involving 728 patients on background therapy of amlodipine $10 \mathrm{mg}$ daily to which ivabradine ( $5 \mathrm{mg}$ bid [232 patients] or $7.5 \mathrm{mg}$ bid [244 patients]) or placebo (252 patients) were added (Figure 5). Compared with placebo, at peak of drug activity (but not at trough) there was a significant increase in total exercise duration in both ivabradine groups $(P=0.005$ and 0.025 for 5 and $7.5 \mathrm{mg}$ respectively); there was also a 
decrease in number of diary-recorded angina attacks and consumption of short-acting nitrates.

\section{Clinical effects: impact on natural history in chronic, stable CAD BEAUTIFUL study}

The association between HR with morbidity/mortality has been found not only in patients with CAD, but also in patients with myocardial infarction (MI), diabetes, hypertension, congestive heart failure, and in free-living populations without known cardiac disorders. ${ }^{47-50}$ In these epidemiological studies, the first of which was published in 1945 involving more than 22,000 American Army Officers, ${ }^{51} \mathrm{HR}$ was directly associated with mortality and, in some cases with major morbid cardiac events. In the morBidity-mortality EvAlUaTion of the $I_{\mathrm{f}}$ inhibitor ivabradine in patients with coronary disease and left-ventricULar dysfunction (BEAUTIFUL study), ${ }^{45}$ it was hypothesized that reducing HR with ivabradine would decrease the composite of mortality, nonfatal acute MI, and hospitalization for heart failure in patients with chronic stable CAD with or without angina. Though often assumed to be true, particularly for $\beta$-blockers, previous to BEAUTIFUL, this hypothesis had been tested only with nicorandil among other anti-anginal, anti-ischemic drugs. ${ }^{52}$ However, while nicorandil reduced cardiac outcome events in the Impact of Nicorandil in Angina (IONA) trial, its anti-anginal dossier is relatively limited. In BEAUTIFUL, in order to maximize the projected number of events in the placebo group (and, thus, minimize the number of patients required in the trial), the high-risk descriptor of left ventricular ejection fraction $\leq 40 \%$ was employed in selecting patients, as well as $\mathrm{HR} \geq 60 \mathrm{bpm}$ at entry (to be consistent with the approved label for the drug and to minimize likelihood of excessive bradycardia with drug) in addition to evidence of CAD by coronary angiography, prior MI, or by any one of several noninvasive CAD tests.

BEAUTIFUL failed to demonstrate an effect of ivabradine on the primary composite endpoint for the study population as a whole. However, in a pre-specified substudy of patients with $\mathrm{HR} \geq 70 \mathrm{bpm}$, ivabradine decreased the incidence of fatal and nonfatal MI $(P=0.001$, relative risk reduction of $36 \%$ ) as well as other secondary endpoints related to active ischemia (eg, coronary revascularization relative risk reduction $30 \%[P=0.016]$ ), while not significantly affecting the primary endpoint. (Though no significant effect was observed for heart failure hospitalizations, this subsequently was studied in another large trial [SHIFT],${ }^{53}$ in which reduction in mortality or hospitalizations due to heart failure were significantly reduced by ivabradine versus placebo on a background of guideline-suggested standard therapies, including $\beta$-blockers.) One reason for the lack of effect of ivabradine on the heart failure outcomes could be that the impact of HR on outcome appears to differ in different conditions, and the BEAUTIFUL population differed from heart failure populations usually employed in clinical trials like SHIFT, and that SHIFT included only patients with HR $\geq 70 \mathrm{bpm}$. Indeed, a subgroup analysis in the placebo cohort showed that for patients with $\mathrm{HR} \geq 70 \mathrm{bpm}$ there was a $53 \%$ increase in hospital admission for heart failure, $34 \%$ excess in risk for cardiovascular death, $46 \%$ increase in hospital admission for MI (fatal and nonfatal) and a 38\% increase in coronary revascularization as compared with patients with

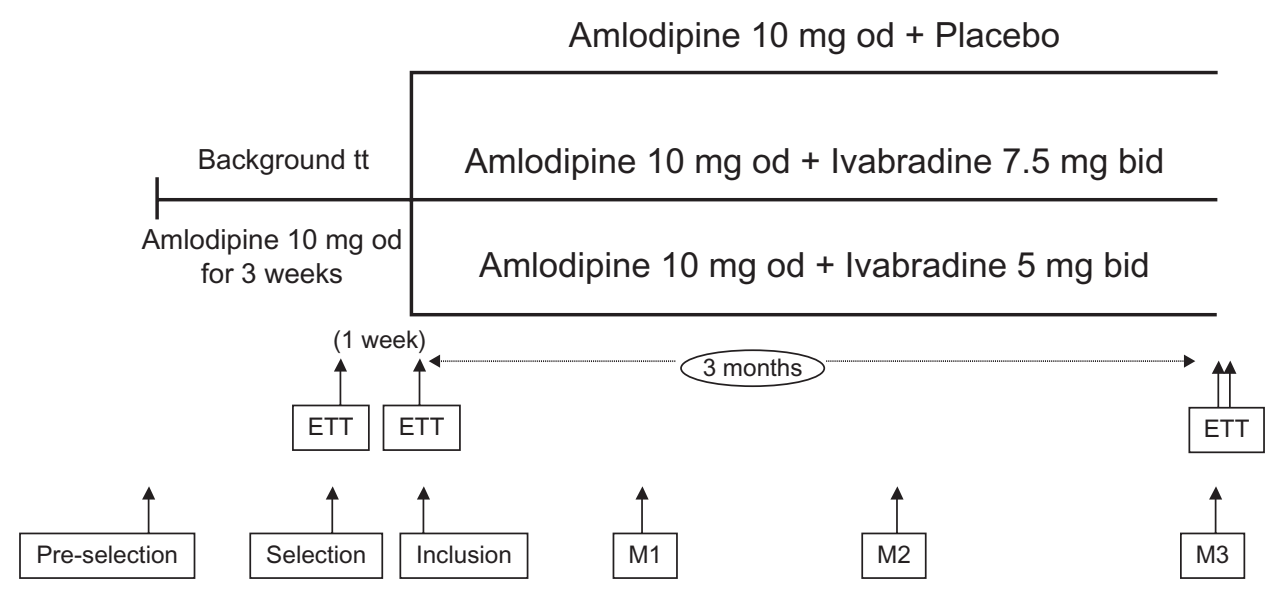

Figure 5 Study design of placebo-controlled clinical trial of ivabradine versus placebo on background of amlodipine for prevention of chronic stable angina $(\mathrm{n}=728$ ). Note: Modified from Tendera et a $\left.\right|^{40}$ with permission from the publisher.

Abbreviations: ETT, exercise tolerance testing; bid, twice daily; M, month; od, once daily; tt, therapy. 
HR $<70$ bpm. The risk for cardiovascular death and admission to hospital for heart failure outcomes was directly proportional to increased HR above $70 \mathrm{bpm}$, whereas the relation was less evident for coronary outcomes. ${ }^{54}$

A post-hoc analysis assessed the effects of ivabradine on the 1507 patients who had entered BEAUTIFUL with CAD, left-ventricular dysfunction, and whose limiting symptom was angina at the time of randomization. ${ }^{55}$ The effects of ivabradine were striking, with a $24 \%$ reduction in the primary endpoint (cardiovascular mortality or hospitalization for fatal and nonfatal $\mathrm{MI}$ or heart failure $[P=0.05$, hazard ratio $=0.76$ versus placebo]). This finding was apparent irrespective of HR at randomization, though it was most striking in patients with $\mathrm{HR} \geq 70 \mathrm{bpm}$ at randomization, who manifested a $31 \%$ reduction in the primary outcome $(P=0.06$, hazard ratio $=0.69)$. Ivabradine reduced hospitalization for MI by $42 \%(P=0.021$, hazard ratio $=0.58)$ in all patients with angina and by $73 \%$ in patients with $\mathrm{HR} \geq 70 \mathrm{bpm}(P=0.002$, hazard ratio $=0.27$ ).

\section{Clinical effects: safety and tolerability}

Throughout its large clinical development program involving almost 20,000 patients and 800 healthy volunteers, ivabradine has demonstrated a very acceptable safety and tolerability profile. ${ }^{41,56}$ The most frequently reported dose-related adverse events have been visual symptoms, the majority being phosphenes that were transient and mild. These symptoms, comprising enhanced brightness in restricted areas of the visual field, were frequently associated with abrupt changes in light intensity. 2,37,41,56 Although the symptom was reported in approximately $15 \%$ of patients in initial trials in which the case report form (CRF) specifically asked for information about such symptoms, the incidence fell to approximately $2 \%$ when the CRF was altered to request information generally about visual symptoms, without specifying symptom type. In any case, these symptoms were dose-related but were well tolerated, causing $<1 \%$ of patients to withdraw from treatment. ${ }^{2,21,37,41}$ This adverse effect has been attributed to the action of ivabradine at the hyperpolarization-activated, cyclic nucleotide-gated HCN channels known to be present in the retina. ${ }^{2,21,22,41}$ The visual symptoms invariably resolved spontaneously during treatment or shortly after treatment withdrawal and, on specialized testing, did not lead to permanent retinal damage. ${ }^{56}$

Unlike $\beta$-blockers, ivabradine does not interfere with respiratory function and has been safely used in patients with asthma, ${ }^{57}$ nor does it impact on peripheral vascular disease. Also, ivabradine has not been associated with detrimental effects on blood glucose, and a glycated hemoglobin level in patients with diabetes differs modestly but significantly from $\beta$-blockers in this regard. ${ }^{58}$

The low incidence of limiting or symptomatic bradycardia (less than $1 \%$ of patients during monotherapy) could be attributed to the use-dependence of ivabradine's action, which limits the risk of excessive bradycardia in patients with initially low HR. ${ }^{39,41}$

Absence of corrected QT interval prolongation from all patients enrolled in the ivabradine development program, throughout the follow-up period, provides strong evidence that ivabradine has no significant direct effect on duration of ventricular repolarization ${ }^{41,59}$ and does not directly cause pro-arrhythmia. However, the best evidence favoring the lack of ivabradine effect on potentially lethal arrhythmogenesis is from the BEAUTIFUL trial, in which there was no evidence of excess mortality with the drug, and from SHIFT, which nominally indicated an ivabradine-related reduction in mortality.

Also, there have been no signs of tolerance or of rebound angina potentiation with the abrupt discontinuation of ivabradine even after prolonged treatment or exposure to high plasma concentrations. , $37,38,42^{2}$

\section{Clinical effects: quality of life, patient satisfaction/acceptability, adherence}

Angina can be frightening to the patient, commonly limits capacity for work and recreation, and is a major cause of debility. Angina affects quality of life in all its dimensions, as demonstrated in the Randomized Intervention Treatment of Angina (RITA) trial. ${ }^{4}$ Beyond the need for hospitalization, many patients with chronic chest pain syndromes are temporarily unable to perform normal activities for hours or days, thereby experiencing a reduced quality of life. In a substantial number of patients, the severity of angina symptoms seriously limits everyday activities, often leading to premature retirement. ${ }^{15,60}$ The RITA-2 trial ${ }^{61}$ sought to evaluate the impact of percutaneous transluminal coronary angioplasty versus medical treatment on self-perceived quality of life among 1018 patients with angina. The angioplasty group had significantly greater improvements in physical functioning, vitality, and general health relatively early ( 3 months and 1 year post procedure), but not at 3 years, perhaps in part due to loss of power owing to crossover from drug to angioplasty. However, 
in this context, the importance of RITA is the marked improvement in perception of quality of life with control of angina: quality-of-life scores were strongly related to breathlessness, angina grade, and treadmill exercise time. Nonetheless, the BARI (Bypass Angioplasty Revascularization Investigation) study $^{62}$ reported that approximately $30 \%$ of patients never return to work after coronary revascularization, and $15 \%-20 \%$ of patients rated their own health fair or poor despite revascularization. Thus, prevention of angina with drugs can be expected to importantly improve quality of life for the millions of patients now afflicted with chronic angina and also to improve patients' satisfaction and adherence to medical therapy.

\section{Conclusion \\ Place in therapy}

$\beta$-blockers, calcium-channel blockers, and long-acting nitrates currently are the cornerstones in prevention and management of stable angina throughout most of the

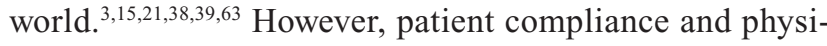
cian use of these treatments may be limited by adverse effects, particularly in the setting of comorbidities, owing to concerns about safety, contraindications, or development of tolerance. ${ }^{2,15,21,38,63}$ Nonetheless, $\beta$-blockers, particularly, have a well established record of effectiveness for preventing angina. Therefore, the risk of adverse effects with $\beta$-blockers must be considered in context of their established benefits in decisions as to their use. ${ }^{15}$ Due to advantages in precautions and side-effects, $\beta$ - 1 selective agents are preferred over nonselective $\beta$-blockers. ${ }^{15}$ Chronic obstructive pulmonary disease and asthma are relative contraindications to $\beta$-blocker use and caution is advised, but some authors believe that $\beta$-blockers at least should be attempted in patients with mild-to-moderate well controlled asthma. ${ }^{64}$ Nevertheless, some patients develop wheezing and bronchospasm with $\beta$-blockers, which then requires dosage reduction or withdrawal of treatment. In addition, $\beta$-blockers can be problematic in patients with peripheral vascular disease or diabetes, while calciumchannel blockers often cause annoying lower extremity edema and constipation, as well as, less commonly, gingival hyperplasia and light-headedness due to peripheral vasodilatation.

Since none of these problems occurs with ivabradine, and in view of the current data on the drug's efficacy and general safety and its demonstrated capacity to add to the benefits of other drugs when used in combination, there appears to be an important clinical role for ivabradine in patients with chronic stable angina. In different subpopulations of patients with stable angina, ivabradine markedly improves all ETT parameters and significantly decreases the number of ambient angina attacks ${ }^{40}$ when given to patients with resting $\mathrm{HR} \geq 60 \mathrm{bpm}$, the criterion for entry into the ivabradine anti-anginal drug trials. In addition, however, the post-hoc angina substudy of BEAUTIFUL suggests another important reason to consider this drug. None of the other most commonly employed anti-anginal, anti-ischemic drugs is known to beneficially affect lethal or major morbid CAD events in patients with angina; indeed, only nicorandil has been studied to assess such effects in this population (with a successful outcome, as noted previously). The apparent life-prolonging and event-reducing benefits of ivabradine in patients with angina, as well as the drug's demonstrated anti-anginal anti-ischemic efficacy, thus provide a basis for strongly considering this drug for treatment of patients with angina. Moreover, though the entire BEAUTIFUL trial, including patients with and without angina, did not meet its prespecified primary endpoint, the substantial reduction in secondary prespecified ischemic events in patients with $\mathrm{HR} \geq 70 \mathrm{bpm}$, including $\mathrm{MI}$ and revascularization, supports the conclusions of the post-hoc analysis. These findings suggest that ivabradine may have a unique role in the armamentarium of physicians treating patients with angina pectoris due to CAD.

Internationally, ivabradine has been approved for marketing for patients with angina by several regulatory agencies. These include the European Agency for Evaluation of Medicinal Products (EMEA), as well as national bodies throughout the world. The drug has never been submitted for review by the FDA because the sponsor lacks an American subsidiary to market the drug, but it is hoped that a submission to the FDA will follow shortly. In addition, several professional societies that issue practice guidelines have endorsed the use of ivabradine. These include the European Society of Cardiology, the guidelines of which were formulated before the completion of the ASSOCIATE study, which recommend ivabradine for the management of stable angina pectoris as an alternative treatment in patients who cannot tolerate $\beta$-blockade, ${ }^{15}$ while the latest statement of the EMEA, updated since ASSOCIATE, also indicates approval as an additive to $\beta$-blockade in patients for whom $\beta$-blockade alone is not sufficient for angina management. ${ }^{36}$ These indications for 
ivabradine use are quite reasonable. However, based on its effectiveness as an anti-anginal, the lack of any data demonstrating superior anti-anginal efficacy of any other drug, its relative safety and its apparent beneficial impact on natural history among patients with angina, it is also reasonable to consider ivabradine as a "first-line" therapy, though such application currently would be considered "off label."

HR reduction is a primary goal for the optimal pharmacological management of chronic stable angina. ${ }^{2,7,39}$ HR is directly associated with risk of cardiovascular death, hospitalization for MI, and coronary revascularization in patients with CAD. ${ }^{45,47} \mathrm{HR}$ reduction with ivabradine, a drug with no other known cardiovascular effects, has resulted in anti-ischemic and anti-anginal efficacy comparable to that of atenolol and to amlodipine and is therefore a valuable therapeutic strategy for chronic stable angina as an alternative or addition to the current pharmacological armamentarium. ${ }^{3,7}$ In addition to its effects on ischemia and angina, ivabradine appears to improve clinical outcomes in patients with limiting angina or in patients with CAD and relatively high HR ( $\geq 70 \mathrm{bpm}) .{ }^{54,55}$ Indeed, from clinical and experimental evidence, ivabradine appears to importantly affect three primary goals of treatment of patients with CAD: control of symptoms, prevention of cardiovascular events, and slowing progression of atherosclerosis. Current safety data show good tolerability with transient visual disturbances and bradycardia as the only potentially important, though relatively infrequent,

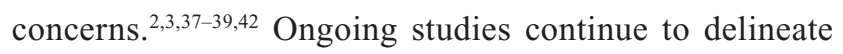
whether pure HR reduction results in decreased mortality/ major morbidity in patients with CAD and other cardiovascular problems. ${ }^{45,47-51,53-55}$

\section{Disclosure}

Dr Borer is a paid consultant to Servier Laboratoires, Neuilly sur Seine, France. However, his work in this study was not supported by Servier but rather by grants from The Howard Gilman Foundation (New York, NY), The Schiavone Family Foundation, White House Station (NJ), The Charles and Jean Brunie Foundation (Bronxville, NY), The American Cardiovascular Research Foundation (New York, NY), The Irving A Hansen Foundation (New York, NY), The Mary AH Rumsey Foundation (New York, NY), The Messinger Family Foundation (New York, NY), The Daniel and Elaine Sargent Charitable Trust (New York, NY), and by much appreciated gifts from Donna and William Acquavella
(New York, NY), and Clyde and Diana Brownstone (New York, NY).

\section{References}

1. Lopez AD, Mathers CD, Ezzati M, Jamison DT, Murray CJ. Global and regional burden of disease and risk factors, 2001: systematic analysis of population health data. Lancet. 2006;367(9524):1747-1757.

2. Sulfi S, Timmis AD. Ivabradine - the first selective sinus node If channel inhibitor in the treatment of stable angina. Int $J$ Clin Pract. 2006;60(2):222-228.

3. Tardif J-C, Ford I, Tendera M, Bourassa MG, Fox K. Efficacy of ivabradine, a new selective If inhibitor, compared with atenolol in patients with chronic stable angina. Eur Heart J. 2005;26(23):2529-2536.

4. Pocock S, Henderson R, Seed P, Treasure T, Hampton JR. Quality of life, employment status, and anginal symptoms after coronary angioplasty or bypass surgery 3-year follow-up in the Randomized Intervention Treatment of Angina (RITA) trial. Circulation. 1996;94(2):135-142.

5. Brorsson B, Bernstein SJ, Brook RH, Werko L. Quality of life of patients with chronic stable angina before and four years after coronary revascularization compared with a normal population. Heart. 2002;87(2):140-145.

6. Mathers CD, Loncar D. Projections of global mortality and burden of disease from 2002 to 2030. PLoS Med. 2006;3(11):e442.

7. Borer JS. Heart rate: from risk marker to risk factor. Eur Heart $J$ Supplements 2008;10(Suppl F):F2-F6.

8. Beere PA, Glagov S, Zarins CK. Retarding effect of lowered heart rate on coronary atherosclerosis. Science. 1984;226(4671):180-182.

9. Persky A, Olsson G, Landou C, deFaire U, Theorell T, Hamstern A. Minimum heart rate and coronary atherosclerosis: independent relations to global severity and rate of progression of angiographic lesions in men with myocardial infarction at a young age. Am Heart $J$. 1992;123(3):609-616.

10. PerskyA, Hamstern A, Lindwall K, Theorell T. Heart rate correlates with severity of coronary atherosclerosis in young post-infarction patients. Am Heart J. 1988;116(5 Pt 1):1369-1373.

11. Heidland UE, Strauer BE. Left ventricular muscle mass and elevated heart rate are associated with coronary plaque disruption. Circulation. 2001;104(13):1477-1482.

12. DiFrancesco D, Camm JA. Heart rate lowering by specific and selective I(f) current inhibition with ivabradine: a new therapeutic perspective in cardiovascular disease. Drugs. 2004;64(16):1757-1765.

13. Rosen MR, Bucchi A, Robinson RB. If modulation: perspectives in clinical medicine. Eur Heart J. 2006;8(Suppl D):3-8.

14. Borer JS. Drug insight: if inhibitors as specific heart-rate-reducing agents. Nat Clin Pract Cardiovasc Med. 2004;1(2):103-109.

15. The Task Force on the Management of Stable Angina Pectoris of the European Society of Cardiology. Guidelines on the management of stable angina pectoris: executive summary. Eur Heart J. 2006; 27(11):1341-1381.

16. Purcell H, Fox K. Selective and specific $I_{\mathrm{f}}$ inhibition: new perspectives. Medicographia. 2005;27(82):51-54.

17. Pepine CJ, Abrams J, Marks RG, Morris JJ, Scheidt SS, Handberg E. Characteristics of a contemporary population with angina pectoris. TIDES Investigators. Am J Cardiol. 1994;74(3):226-231.

18. DiFrancesco D. Pacemaker mechanisms in cardiac tissue. Annu Rev Physiol. 1993;55:455-472.

19. DiFrancesco D. Serious working of the funny current. Prog Biophys Mol Biol. 2006;90(1-3):13-25.

20. Ferrari R, Campo G, Gardini E, Pasanisi G, Ceconi C. Specific and selective $I_{\mathrm{f}}$ inhibition: expected clinical benefits from pure heart rate reduction in coronary patients. Eur Heart J Supplements. 2005; 7(Suppl H):H16-H21.

21. Shattock M, Camm AJ. Pure heart rate reduction: the $I_{\mathrm{f}}$ channels from discovery to therapeutic target. Br J Cardiol. 2006;13(1): $27-35$. 
22. Saha M, Marber MS. If at first you don't succeed try ... a new target in the treatment of angina. Eur Heart J. 2005;26(23):2482-2483.

23. DiFrancesco D. $I_{\mathrm{f}}$ inhibition: a novel mechanism of action. Eur Heart J Supplements. 2003;5(Suppl G):G19-G25.

24. Brown HF, DiFrancesco D, Noble SJ. How does adrenaline accelerate the heart? Nature. 1979;280(5719):235-236.

25. Baruscotti M, Bucchi A, Difrancesco D. Physiology and pharmacology of the cardiac pacemaker ("funny") current. Pharmacol Ther. 2005;107(1):59-79.

26. DiFrancesco D, Ducouret P, Robinson RB. Muscarinic modulation of cardiac rate at low acetylcholine concentrations. Science. 1989;243(4891):669-671.

27. DiFrancesco D, Tortora P. Direct activation of cardiac pacemaker channels by intracellular cyclic AMP. Nature. 1991;351(6322):145-147.

28. DiFrancesco D. The onset and autonomic regulation of cardiac pacemaker activity: relevance of the f current. Cardiovasc Res. 1995;29(4):449-456.

29. DiFrancesco D. The pacemaker current If plays an important role in regulating $\mathrm{SA}$ node pacemaker activity. Cardiovasc Res. 1995;30(2):307-308.

30. Barbuti A, Baruscotti M, DiFrancesco D. The pacemaker current: from basics to the clinics. J Cardiovasc Electrophysiol. 2007; 18(3):342-347.

31. Robinson RB, Siegelbaum SA. Hyperpolarization-activated cation currents: from molecules to physiological function. Annu Rev Physiol. 2003;65:453-480.

32. César LAM. $I_{\mathrm{f}}$ current and heart rate control. Arq Bras Cardiol. 2007;88(4):e96-e99.

33. Bucchi A, Tognati A, Milanesi R, Baruscotti M, DiFrancesco D. Properties of ivabradine-induced block of $\mathrm{HCN} 1$ and $\mathrm{HCN} 4$ pacemaker channels. J Physiol. 2006;572(Pt 2):335-346.

34. Bucchi A, Baruscotti M, DiFrancesco D. Current-dependent block of rabbit sino-atrial node $I_{\mathrm{f}}$ channels by ivabradine. J Gen Physiol. 2002;120(1):1-13.

35. DiFrancesco D. Cardiac pacemaker I(f) current and its inhibition by heart rate-reducing agents. Curr Med Res Opin. 2005;21(7):1115-1122.

36. European Medicines Agency. Procoralan: EPAR Product Information: Annex 1; Summary of Product Characteristics. London: European Medicines Agency; 2009 [updated June 8, 2011]. Available from: http://www. ema.europa.eu/docs/en_GB/document_library/EPAR_-_Product_Information/human/000597/WC500043590.pdf. Accessed Aug 19, 2011.

37. Borer JS, Fox K, Jaillon P, Lerebours G. Antianginal and antiischaemic effects of ivabradine, an If inhibitor, in stable angina: a randomized, double-blind, multicentred, placebo-controlled trial. Circulation 2003;107(6):817-823.

38. López-Bescós L, Filipova S, Martos R. Long-term safety and efficacy of ivabradine in patients with chronic stable angina. Cardiology. 2007;108(4):387-396.

39. Tardif JC. Ivabradine $I_{\mathrm{f}}$ inhibition in the management of stable angina pectoris and other cardiovascular diseases. Drugs Today. 2008;44(3):171-181.

40. Tendera M, Borer JS, Tardif JC. Efficacy of I(f) inhibition with ivabradine in different subpopulations with stable angina pectoris. Cardiology. 2009;114(2):116-125.

41. Savelieva I, Camm AJ. $I_{\mathrm{f}}$ inhibition with ivabradine electrophysiological effects and safety. Drugs Safety. 2008;31(2):95-107.

42. Ruzyllo W, Tendera M, Ford I, et al. Antianginal efficacy and safety of ivabradine compared with amlodipine in patients with stable effort angina pectoris: a 3 month randomised, double-blind, multicentre, noninferiority trial. Drugs. 2007;67(3):393-405.

43. Steg PG, Tchetche D. Pharmacologic management of stable angina: role of ivabradine. Eur Heart J Supplements. 2006;8(Suppl D):D16-D23.

44. Tardiff JC, Ponikowski P, Kahan T. Efficacy of the $I_{\mathrm{f}}$ current inhibitor ivabradine in patients with chronic stable angina receiving beta-blocker therapy: a 4-month, randomized, placebo-controlled trial. Eur Heart J. 2009;30(5):540-548
45. Fox K, Ford I, Steg PG, Tendera M, Ferrari R. Ivabradine for patients with stable coronary artery disease and left-ventricular systolic dysfunction (BEAUTIFUL): a randomised, double-blind, placebo-controlled trial. Lancet. 2008;372(9641):807-816.

46. Koester R, Kaehler J, Ebelt H, Soeffker G, Werdan K, Meinertz T. Ivabradine in combination with beta-blocker therapy for the treatment of stable angina pectoris in every day clinical practice. Clin Res Cardiol. 2010;99(10):665-672.

47. Diaz A, Bourassa MG, Guertin MC, Tardif JC. Long-term prognostic value of resting heart rate in patients with suspected or proven coronary artery disease. Eur Heart J. 2005;26(10):967-974.

48. Jouven X, Empana JP, Schwartz PJ, Desnos M, Courbon D, Ducimetiere P. Heart-rate profile during exercise as a predictor of sudden death. $N$ Engl J Med. 2005;352(19): 1951-1958.

49. Cucherat M. Relationship between heart rate lowering and benefits on cardiac and sudden death observed with beta-blockers in post MI patients. A meta-regression of randomized clinical trials. Eur Heart J. 2007;28(24):3012-3019.

50. Dyer AR, Persky V, Stamler J, et al. Lindberg HA. Heart rate as a prognostic factor for coronary heart disease and mortality: findings in three Chicago epidemiologic studies. Am J Epidemiol. 1980;112(6):736-749.

51. Levy RL, White PD, Stroud WD, Hillman CC: transient tachycardia: prognostic significance alone and in association with transient hypertension. JAMA. 1945;129(9):585-588.

52. IONA Study Group. Effect of nicorandil on coronary events in patients with stable angina: the Impact of Nicorandil in Angina (IONA) randomised trial. Lancet. 2002;359(9314): 1269-1275.

53. Swedberg K, Komajda M, Böhm M, et al. Ivabradine and outcomes in chronic heart failure (SHIFT): a randomised placebo-controlled study. Lancet. 2010;376(9744):875-885.

54. Fox K, Ford I, Steg PG, Tendera M, Robertson M, Ferrari R. Heart rate as a prognostic risk factor in patients with coronary artery disease and left ventricular systolic dysfunction (BEAUTIFUL): a subgroup analysis of a randomised controlled trial. Lancet. 2008;372(9641): $817-821$

55. Fox K, Ford I, Gabriel PH, Tendera M, Robertson M, Roberto F. Relationship between ivabradine treatment and cardiovascular outcomes in patients with stable coronary artery disease and left ventricular systolic dysfunction with limiting angina: a subgroup analysis of the randomized controlled BEAUTIFUL trial. Eur Heart J. 2009;30(19): 2337-2345.

56. Tardif J-C. Ivabradine in clinical practice: benefits of $I_{\mathrm{f}}$ inhibition. Eur Heart J Suppl. 2005;7(Suppl H):H29-H32.

57. Babu S, Gadzik F, Holgate S. Absence of respiratory effects with ivabradine in patients with asthma. Br J Clin Pharmacol. 2008;66(1):96-101.

58. Borer JS, Tardif JC. Efficacy of ivabradine, a selective $I_{\mathrm{f}}$ inhibitor, in patients with chronic stable angina pectoris and diabetes mellitus. Am J Cardiol. 2010;105(1):29-35.

59. Savelieva I, Camm AJ. Absence of direct effects of the $I_{\mathrm{f}}$ current blocker ivabradine on ventricular repolarization: analysis based on a population heart rate correction formula. J Am Coll Cardiol. 2005;45(Suppl):95A.

60. Gibbons RJ, Abrams J, Chatterjee K, et al. ACC/AHA 2002 guideline update for the management of patients with chronic stable angina summary article: a report of the American College of Cardiology/ American Heart Association Task Force on Practice Guidelines (Committee on the Management of Patients with Chronic Stable Angina). Circulation. 2003;107(1):149-158.

61. Pocock S, Henderson R, Clayton T, Lyman G, Chamberlain D. Quality of life after coronary angioplasty or continued medical treatment for angina: three-year follow-up in the RITA-2 trial. J Am Coll Cardiol. 2000;35(4):907-914. 
62. Writing Group for the Bypass Angioplasty Revascularization Investigation (BARI) Investigators. Five-year clinical and functional outcome comparing bypass surgery and angioplasty in patients with multivessel coronary disease: a multicenter randomized trial. JAMA. 1997;277(9):715-721.

63. Parker JO. Chronic angina pectoris: inadequacies of current therapy. Am J Geriatr Cardiol. 2004;13(5):261-266.
64. Ashrafian, H, Violaris AG. Beta-blocker therapy of cardiovascular diseases in patients with bronchial asthma or COPD: the pro viewpoint. Prim Care Respir J. 2005;14(5):236-241.

\section{Publish your work in this journal}

Research Reports in Clinical Cardiology is an international, peerreviewed, open access journal publishing original research, reports, editorials, reviews and commentaries on all areas of cardiology in the clinic and laboratory. The manuscript management system is completely online and includes a very quick and fair peer-review system.
Visit http://www.dovepress.com/testimonials.php to read real quotes from published authors. 